\title{
Specific Amplification by PCR of Rearranged Genomic Variable Regions of Immunoglobulin Genes from Mouse Hybridoma Cells
}

\author{
José Berdoz, ${ }^{1}$ Thomas P. Monath, ${ }^{2}$ and Jean-Pierre Kraehenbuhl ${ }^{1}$
}

${ }^{1}$ Swiss Institute for Experimental Cancer Research and Institute of Biochemistry, University of Lausanne, Switzerland; ${ }^{2}$ OraVax Inc., Cambridge, Massachusetts 02139

\begin{abstract}
We have designed a novel strategy for the isolation of the rearranged genomic fragments encoding the $L-V_{H^{\prime}}-D-J_{H}$ and $L-V_{K / \lambda}-J_{K / \lambda}$ regions of mouse immunoglobulin genes. This strategy is based on the PCR ampliffcation of genomic DNA from mouse hybridomas using multiple specific primers chosen in the 5 -untranslated region and in the intron downstream of the rearranged $J_{H} / J_{\kappa / \lambda}$ sequences. Variable regions with intact coding sequences, including fulllength leader peptides (L) can be obtained without previous DNA sequencing. Our strategy is based on a genomic template that produces fragments that do not need to be adapted for recombinant antibody expression, thus facilitating the generation of chimeric and isotypeswitched immunoglobulins.
\end{abstract}

$\mathbf{T}$ he development of mouse hybridoma technology has allowed the production of antibodies specific to a wide range of antigens. Mouse monoclonal antibodies (mAbs) have been used extensively for diagnosis, and some have proven useful for treatment of human diseases. Generally, murine antibodies are cleared rapidly from the circulation and often do not interact effectively with the host immune system. Moreover, administration of murine antibodies to humans often induces a human anti-mouse antibody (HAMA) response after single or repeated treatments. ${ }^{(1)}$ Attempts to replace murine mAbs have been limited by the difficulties in generating human hybridomas, which are generally unstable and secrete low amounts of antibodies (frequently IgMs). Thus, considerable efforts have been made to render murine mAbs more similar to those of the human host. Alternatives to human hybridoma-derived antibodies have been developed in which most of the rodentderived sequences of mouse immunoglobulins are replaced with sequences derived from human immunoglobulins. Two approaches have been used: (1) chimeric mAbs, in which the xeno-V regions are combined with human constant domain regions (CDRs); ${ }^{(2,3)}$ and (2) humanized mAbs, in which only the xeno-CDRs are linked onto human sequences. ${ }^{(4,5)}$ These engineered antibodies retain their target specificity and show reduced HAMA responses when injected into patients. ${ }^{(6,7)}$ In addition, the correct effector function of the antibody as required for certain clinical applications can be obtained by selecting the corresponding immunoglobulin isotype.

In spite of these advances, cloning of the variable region sequences has been a limiting step in the rapid construction of chimeric and isotype-switched antibody molecules. Amplification by PCR of the immunoglobulin heavy- and light-chain variable regions has facilitated this step greatly. However, the high degree of DNA sequence polymorphism in the leader and variable sequences of both heavy and light chain genes has required the preparation of complex sets of degenerate primers. ${ }^{(8-11)}$ These primers are usually within the first framework of the variable region (FR1), and in a few cases in the leader peptide sequence (L). The 3 ' primers are usually within framework 4 (FR4), which displays a limited polymorphism, or in the constant region, in which conserved, isotype-specific sequences can be identified easily. Therefore, most of the current PCR amplification reactions of the immunoglobulin $\mathrm{H}$ and $\kappa / \lambda$ chain variable regions generate fragments containing incomplete $V_{H}$ and $V_{L}$ sequences, linked or not linked to part of the constant region. When cloned into cassette expression vectors, such fragments generate chimeric immunoglobulins directly when PCR primers are within the FR1 and FR4 regions. Additional modifications such as the reconstruction of the original splice donor site of the $\mathrm{V}$ region or the mutagenesis of sites in the $\mathrm{V}$ and $\mathrm{C}$ regions that create $\mathrm{a}$ common restriction sequence are required before cloning when the fragments contain $\mathrm{C}$ regions. ${ }^{(12,13)}$ Other disadvantages of the methods currently 
in use arise from the observation that although complex sets of $5^{\prime}$ and $3^{\prime}$ primers have been designed, they do not always match the DNA template completely. ${ }^{(14,15)}$ Native sequences of the immunoglobulin heavy and light chains may therefore be altered in the FR1 and/ or in the FR4 regions during the amplification process. This can be a problem because modifications of the amino-terminal structure have been shown to reduce drastically the affinity of the immunoglobulin for its antigen. This is particularly true for the light-chain variable region, in which the amino acid at position 2 is part of the predicted canonical structure for CDR1. ${ }^{(16)}$ Moreover, expression levels of the recombinant antibody may be altered when mutations occur in the leader peptide.

Other current technologies such as inverse or anchored PCR circumvent some of the problems described above. For example, one can determine the $5^{\prime}$ and 3 ' sequences of the variable regions, and therefore PCR primers that match the template exactly can be prepared. ${ }^{(17,18)}$ Variable regions with unaltered amino-terminal sequences can be generated. However, these procedures are time-consuming.

If DNA sequences of the $V_{H}$ and $V_{L}$ genomic regions from the parental hybridoma are known, including the 5 '-untranslated regions and the introns downstream of the rearranged J segments, PCR-amplified fragments can be inserted into expression vectors within noncoding sequences. ${ }^{(19)}$ This procedure generates complete, original variable regions that can be linked without modifications to any other constant region, provided that the reading frame of the $V$ region is maintained.

We have developed a general method for PCR amplification of the rearranged genomic fragments encoding full-length variable regions of mouse immunoglobulin genes. This method does not require previous DNA sequencing. Amplified fragments can be characterized readily using a set of J-specific oligonucleotide probes that are used also for typing of the $\mathrm{V}_{\mathrm{H}}-\mathrm{D}-\mathrm{J}_{\mathrm{H}}$ and $\mathrm{V}_{\mathrm{L}}-\mathrm{J}_{\mathrm{L}}$ rearrangements on hybridoma RNA.

\section{MATERIALS AND METHODS Sources of DNA and RNA}

Mouse hybridoma cells HNK-20 (IgA, к) and $3 \mathrm{G} 3(\operatorname{IgM}, \lambda)$ were obtained from OraVax, Inc. (Cambridge, MA) and from American Type Culture Collection (ATCC, Rockville, MD) no. HB8516, respectively. Genomic DNA was prepared as described. ${ }^{(20)}$ Total RNA was prepared from frozen cell pellets according to the guanidine/cesium chloride method. ${ }^{(21,22)}$

\section{Synthesis of Oligonucleotide Primers and Probes}

Oligonucleotides were synthesized by the $\beta$-cyanoethyl phosphoramidite method and purified by reverse-phase HPLC (MWG-Biotech, Ebersberg, Germany).

\section{DNA Amplification by PCR}

In vitro DNA amplification was performed in $100-\mu$ l final volume in a Perkin-Elmer 9600 thermal cycler (Norwalk, CT). Reagents were added to the reaction to yield the following final composition: $10 \mathrm{~mm}$ Tris- $\mathrm{HCl}(\mathrm{pH} 8.3)$ at $25^{\circ} \mathrm{C} ; 50 \mathrm{~mm}$ $\mathrm{KCl} ; 2.5 \mathrm{~mm} \mathrm{MgCl}_{2} ; 0.001 \%$ gelatin (Sigma, cat. no. G2500, St. Louis, MO); 200 mм dNTP; $150 \mathrm{~nm}$ of each amplification primer; $1 \mu \mathrm{g}$ of genomic DNA; and 2.5 units of AmpliTaq DNA polymerase (Perkin-Elmer, Norwalk, CT). The cycling profile was as follows: $5 \mathrm{~min}$ at $94^{\circ} \mathrm{C}, 3$ cycles of $1 \mathrm{~min}$ at $94^{\circ} \mathrm{C}, 1 \mathrm{~min}$ at $50^{\circ} \mathrm{C}, 1 \mathrm{~min}$ at $72^{\circ} \mathrm{C}, 27$ cycles of $1 \mathrm{~min}$ at $94^{\circ} \mathrm{C}, 1 \mathrm{~min}$ at $62^{\circ} \mathrm{C}, 1 \mathrm{~min}$ at $72^{\circ} \mathrm{C}$, and one final incubation at $72^{\circ} \mathrm{C}$ for 10 $\min$.

\section{Northern and Southern Blots}

Total RNA $(10 \mu \mathrm{g})$ was denatured with glyoxal and subjected to electrophoresis on an agarose gel. ${ }^{(23)}$ The sample was transferred onto a GeneScreen Plus membrane (Du Pont, Wilmington, DE) according to the procedure described by the manufacturer. Products of PCR amplification of genomic DNA prepared from hybridoma cells were analyzed on agarose gels and transferred onto a GeneScreen Plus membrane as described. ${ }^{(24)}$

Northern and Southern blots were hybridized under identical conditions with ${ }^{32}$ P-labeled oligonucleotide probes specific for each of the $J_{\lambda}, J_{\kappa}$ and $J_{H}$ segments. Temperatures [melting temperature $\left.\left(T_{\mathrm{m}}\right)\right]$ of prehybridization and hybridization were at $T_{\mathrm{m}}-4^{\circ} \mathrm{C}$, and washings at $T_{\mathrm{m}}-2^{\circ} \mathrm{C}$. The $\mathrm{T}_{\mathrm{m}} \mathrm{s}$ of the oligonucleotides were estimated by the formula: $T_{\mathrm{m}}=4$ (number of $\mathrm{G}+\mathrm{C}$ ) +2 (number of $\mathrm{A}+\mathrm{T}$ ). The $T_{\mathrm{m}} \mathrm{s}$ of the J-specific oligonucleotide probes are as follows: $\mathrm{J}_{\lambda 1}=58^{\circ} \mathrm{C} ; \mathrm{J}_{\lambda 2}=62^{\circ} \mathrm{C}$; a)

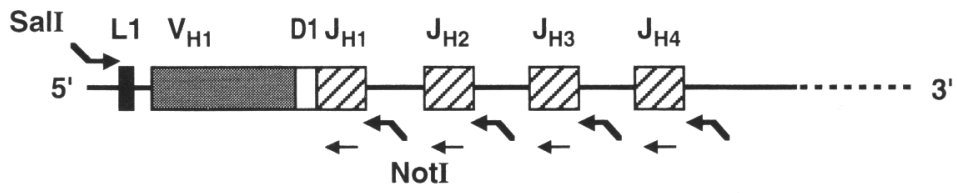

b)

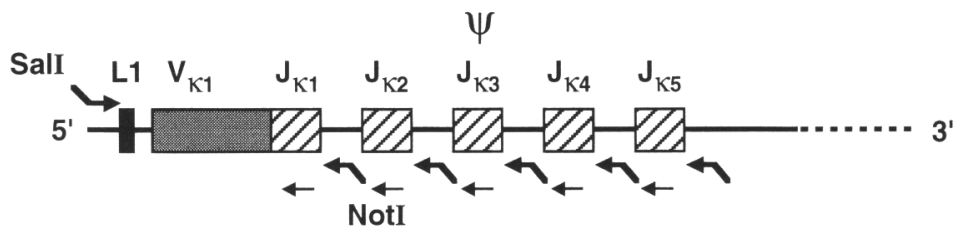

c)

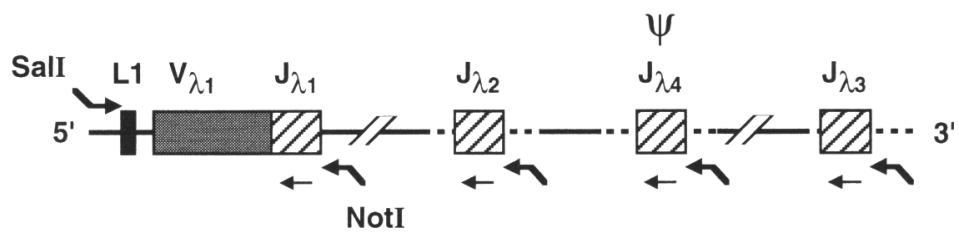

FIGURE 1 Map of the rearranged genes encoding the variable regions of mouse immunoglobulin heavy $(a), \kappa(b)$, and $\lambda(c)$ chains showing the location of the PCR primers and probes used for the amplification of $V_{H}$ and $V_{L}$ regions. Pseudogenes are indicated by $\Psi$. Bold, bent arrows indicate the PCR primers located in the 5 '-untranslated regions and in the introns downstream of the J segments. SalI and NotI restriction sites have been added at the $5^{\prime}$ ends of the PCR primers. Straight arrows below the J segments indicate the oligonucleotide probes used in Northern and Southern blot hybridization. 
$\mathrm{J}_{\lambda 3}=60^{\circ} \mathrm{C} ; \mathrm{J}_{\lambda 4}=58^{\circ} \mathrm{C} ; \mathrm{J}_{\kappa 1}=64^{\circ} \mathrm{C} ; \mathrm{J}_{\kappa 2}=64^{\circ} \mathrm{C} ;$ $\mathrm{J}_{\kappa 3}=56^{\circ} \mathrm{C} ; \quad \mathrm{J}_{\kappa 4}=64^{\circ} \mathrm{C} ; \quad \mathrm{J}_{\kappa 5}=62^{\circ} \mathrm{C}$; $\mathrm{J}_{\mathrm{H} 1}=68^{\circ} \mathrm{C} ; \mathrm{J}_{\mathrm{H} 2}=64^{\circ} \mathrm{C} ; \mathrm{J}_{\mathrm{H} 3}=64^{\circ} \mathrm{C} ; \quad$ and $\mathrm{J}_{\mathrm{H} 4}=60^{\circ} \mathrm{C}$.

Prehybridization was done for 3-4 hr in $2 \times$ SSC, $5 \times$ Denhardt's solution, $0.1 \%$ SDS, and 5 mM EDTA. Hybridization was for $14-18 \mathrm{hr}$ in $5 \times$ SSC, $10 \times$ Denhardt's solution, $20 \mathrm{~mm}$ sodium phosphate buffer ( $\mathrm{pH} 7.0$ ), 7\% SDS, $100 \mu \mathrm{g} / \mathrm{ml}$ of denatured herring sperm DNA, and ${ }^{32} \mathrm{P}$ labeled oligonucleotide $\left(10^{6} \mathrm{cpm} / \mathrm{ml}\right)$. The membranes were then washed twice for $30 \mathrm{~min}$ in $3 \times$ SSC, $10 \times$ Denhardt's solution, $5 \%$ SDS, $70 \mathrm{~mm}$ sodium phosphate buffer ( $\mathrm{pH} \mathrm{7.0)}$; and twice for 30 min in $1 \times$ SSC and $1 \%$ SDS.

As an internal control for total RNA loading, Northern blots were hybridized with a glyceraldehyde-3-phosphate-dehydrogenase cDNA probe. ${ }^{(25)}$ Prehybridization was done for $3-5 \mathrm{hr}$ at $42^{\circ} \mathrm{C}$ in $50 \%$ deionized formamide, $5 \times$ Denhardt's solution, $5 \times$ SSPE [30 $\times$ SSPE $=4.5 \mathrm{M} \mathrm{NaCl}, 0.3 \mathrm{M} \mathrm{NaH}_{2} \mathrm{PO}_{4}, 30$ mM EDTA (pH 7.7)], 1\% SDS, and 200 $\mu \mathrm{g} / \mathrm{ml}$ of denatured salmon sperm DNA. Hybridization was for $14-18 \mathrm{hr}$ at $42^{\circ} \mathrm{C}$ in the same mixture with ${ }^{32} \mathrm{P}$-labeled cDNA probe $\left(10^{6} \mathrm{cpm} / \mathrm{ml}\right)$. The membranes were then washed twice for $5 \mathrm{~min}$ in $2 \times$ SSPE at room temperature, once for $15 \mathrm{~min}$ in $2 \times$ SSPE, and $0.5 \%$ SDS at $65^{\circ} \mathrm{C}$, and once for $15 \mathrm{~min}$ in $0.5 \times \mathrm{SSPE}$ at $65^{\circ} \mathrm{C}$.

\section{DNA Sequencing}

DNA sequences were derived by subcloning the $\mathrm{L}-\mathrm{V}_{\mathrm{H}}-\mathrm{D}-\mathrm{J}_{\mathrm{H}}$ and $\mathrm{L}-\mathrm{V}_{\mathrm{L}}-\mathrm{J}_{\mathrm{L}}$ PCR fragments in M13mp18 or in the expression vector $\mathrm{pING}{ }^{(16)}$ followed by sequencing using the dideoxy chain termination method. (26)

\section{RESULTS AND DISCUSSION \\ Design of Primers for Mouse $V_{H}$ and $V_{L}$ Amplification}

Because most strategies used to amplify mouse $V_{H}$ and $V_{L}$ genes generate fragments that are truncated or that may contain mutations in their $5^{\prime}$ and/or $3^{\prime}$ ends, we have designed new sets of $5^{\prime}$ and $3^{\prime}$ PCR primers that preserve the entire Fv sequences. These primers were chosen in the $5^{\prime}$-untranslated region and in the intron downstream of the rearranged J segment of the $\lambda, \kappa$, and heavychain genes (Fig. 1).
A) PCR primers for $\mathrm{V} \lambda$ :

(a) 5 ' $\lambda 1$ : GATCGTCGACCITGGTTTGTGAATTATG (a) $5 \lambda 2$ : GATCGTCGACAGTAGTACCTGCATTATG 3' $\lambda 1$ : GATCGCGGCCGCAAAGGAGGIAGGAGTIAC 3 ' $\lambda 2$ : ATCAGCGGCCGCAAGAAGCATTAAAGCCAC $32 \lambda$ : ATCAGCGGCCGCAAGAAGCTTTGAAACTAC

B) PCR primers for $\mathrm{V} \kappa$ :

(a) 5 ' 11 : GATCGTCGACAAATTCAAAG/TACAA/CAATG (f) 5' $\mathbf{2}$ : GATCGTCGACAAGACTCAGCCTGACATG (a) $5 * 3$ : GATCGTCGACAAGTTCAAAGACAAAATG (d) 5' $\mathbf{k}$ : GATCGTCGACAGACTCAGCCTIGACATG (h) 5 ' 5 : GATCGTCGACAGCAGGGGGAGCAGGATG (b) 5' 6 : GATCGTCGACAGGGAAAGTTTGAAGATG (d) 5' $\mathrm{k} 7$ : GATCGTCGACATACATCAGACCAGCATG (d) 5' $\mathrm{K} 8$ : GATCGTCGACATCTAGC/TTCTCAGAGATG (f) $5 \mathrm{k} \cdot \mathrm{g}:$ GATCGTCGACATGCATCACACCAGCATG (d) 5'k10: GATCGTCGACCACCAAGTTCTCAGAATG (h) 5'K11: GATCGTCGACCAGAGCAGCAGGGACATG (h) 5'k12: GATCGTCGACCAGGGACAAGTGGGAATG (e) 5'K13: GATCGTCGACCATTCAGAACTCAGCATG (h) 5'K14: GATCGTCGACGCGAGTCAGACCAGCATG (b) 5'k15: GATCGTCGACGGACACAGTTTAGATATG (i) 5'k16: GATCGTCGACGGACTCAGCATGGACATG (e) 5'x17: GATCGTCGACGGAGACGTTGTAGAAATG (f) 5'k18: GATCGTCGACGGATACACCATCAGCATG (i) 5'א19: GATCGTCGACGGCAAA/GGGCATCAAGATG (i) 5'א20: GATCGTCGACGGCAGG/TGGA/GAGCAAGATG (g) 5'k21: GATCGTCGACGGTCACAGCACAAACATG (g) 5' '22: GATCGTCGACGGTTGCCTCCTCAAAATG (b) 5'K23: GATCGTCGACGTTCATTTCCTCAAAATG (a) 5' 2 24: GATCGTCGACTATCAAGTTCICAGAATG (c) 5'K25: GATCGTCGACTCTCAAGTTCTCAGAATG (a) 5' $\mathrm{k}$ 6: GATCGTCGACTCTTGTGAATTAATCATG (c) 5' 27: GATCGTCGACTGAAAACACACAGACATG (c) 5' 28 : GATCGTCGACTGATAAAGCCAAGGAATG (e) 5'K29: GATCGTCGACTGATCACACACAGA/TCATG (g) 5' 30: GATCGTCGACTTCCAGCTCTCAGAGATG 3'k1 : ATCAGCGGCCGCAGAGAG/CTTTGGATTCTAC 3 ' $k 2$ : ATCAGCGGCCGCAAGAGTTGAGAAGACTAC 3' 3 : ATCAGCGGCCGCAGTTGAGCAAAAATGTAC 3 'K4 : ATCAGCGGCCGCAAATGAGCAAAAA/GTCTAC $3 \mathrm{k}$ : ATCAGCGGCCGCAAGATGAGAAAAGTGTAC

D) $\mathrm{J} \lambda$ oligo probes :

$\mathrm{J} \lambda 1$ : GTCAGTTTGGTTCCTCCAC J $\lambda 2$ : GTGACCTTGGTTCCACCGC J $\lambda 3$ : GTGACCTTGGTTCCACTGC $\mathrm{J} \lambda \mathbf{4}$ : GTCAATCTGGTTCCACCTC

F) JH oligo probes :

JH1 : GACCGTGGTCCCTGCGCCC JH2 : GAGAGTGGTGCCTTGGCCC JH3 : GACCAGAGTCCCTTGGCCC JH4 : GACTGAGGTTCCTTGACCC

FIGURE 2 Nucleotide sequences of the PCR primers and probes used for the amplification of mouse $V_{\lambda}, V_{\kappa}$, and $V_{H}$ regions. Sall and NotI sites are in bold. (I) Inosine. The $5^{\prime}$ primers for the PCR amplification of the variable regions were grouped according to melting ternperature, and the groups are designated $(a-l)$ as indicated by the letters preceding each entry. (j) 5'H1 : GATCGTCGACACACAGACTCACACCATG (g) 5'H2 : GATCGTCGACACACAGGACCTCACCATG (j) 5'H3 : GATCGTCGACACACAGGATCTCACCATG (g) 5'H4 : GATCGTCGACACACAGGGCATTGCCATG (b) 5'H5 : GATCGTCGACACACTGACTCAAAACATG (c) 5'H6: GATCGTCGACACACTGACTCAAACCATG (j) 5'H7 : GATCGTCGACACACTGACTCACACCATG (j) 5'H8 : GATCGTCGACACACTGACTCCAACCATG (c) 5'H9 : GATCGTCGACACACTGACTCTAACCATG (k) 5'H10: GATCGTCGACACACTGACTCTCACCATG (c) 5'H11: GATCGTCGACACACTGACTTTCACCATG (b) 5'H12: GATCGTCGACACATAGACTCTAACCATG (b) 5'H13: GATCGTCGACACATTGACTCAAACCATG (g) 5'H14: GATCGTCGACAGCCTCCATCAGAGCATG (e) 5'H15: GATCGTCGACAGCCTCCGTCAGAGCATG (a) 5'H16: GATCGTCGACATTATAACATTGAACATG (b) 5'H17: GATCGTCGACCAAGTCTTAGACATCATG (k) 5'H18: GATCGTCGACCACACATCCCTTACCATG (h) 5'H19: GATCGTCGACCACAGACACCTCACCATG (e) 5'H20: GATCGTCGACCACAGACCA/CCTCACCATG (k) 5'H21: GATCGTCGACCACAGACCTGTCAACATG (h) 5'H22: GATCGTCGACCACAGACCTGTCACCATG (e) 5'H23: GATCGTCGACCACGGAACCCTCACCATG (f) 5'H24: GATCGTCGACCACGGACCCCTCACCATG (f) 5'H25: GATCGTCGACCACGGACCCCTCACGATG (k) 5'H26: GATCGTCGACCACTCGACTCTAACCATG (h) 5'H27: GATCGTCGACCACTGGTGTGCAGTCATG (a) 5'H28: GATCGTCGACCACTTCTTAGACATCATG (f) 5'H29: GATCGTCGACCAGAGTCCACTCA/GCCATG (l) 5'H30: GATCGTCGACCCTGTCACTGACTTCATG (c) 5'H31a:GATCGTCGACCTCAAGGTCCTTACAATG (i) 5'H31b:GATCGTCGACCTCCAGGTCCTTACAATG (i) 5'H32: GATCGTCGACCTCAGTCCTGTCACCATG (i) 5'H33: GATCGTCGACCTCAGTCCTGTCACTATG (i) 5'H34: GATCGTCGACGCAGAGGACCTCACAATG (d) 5'H35: GATCGTCGACGCCTTTACAGACTTCATG (f) 5'H36: GATCGTCGACGGACCTCACCATGGGATG (i) 5'H37: GATCGTCGACGGGTGTTGCCTAAGGATG (d) 5'H38: GATCGTCGACGGTGTA/TGCCTAAAAGATG (a) 5'H39: GATCGTCGACGGTGTTGCCTAAAGGATG (a) 5'H40: GATCGTCGACGTTGTAGCCTAAAAGATG (d) 5'H41: GATCGTCGACTCAGTCCTTGTCACTATG 3'H1 : ATCAGCGGCCGCAAAGAAAAAAGCCAGCTTAC 3'H2 : ATCAGCGGCCGCGAGGTTT/GTAAGGACTCAC 3'H3a: ATCAGCGGCCGCGGAGAAA/GTTAGGACTCAC 3'H3b: ATCAGCGGCCGCGGAGAAGT/GTAGGACTCAC 3'H4 : ATCAGCGGCCGCTGGAGAGGCCATTCTTAC

E) JK oligo probes :

Jк1 : GTGCCTCCACCGAACGTCC JK2 : GTCCCCCCTCCGAACGTGT JK3 : GTCCCATCACTGAATGTGA JK4 : GTCCCCGAGCCGAACGTGA J 5 : GTCCCAGCACCGAACGTGA 
DNA sequence comparisons of the 5'untranslated region and of the intron downstream of the J segments were performed using data from Kabat, ${ }^{(27)}$ and from the GenBank and European Molecular Biology Laboratory (EMBL) libraries. Sequences were analyzed with the Genetics Computer Group (GCG, Madison, WI) package programs. Alignment of the DNA sequences of the 5'-untranslated region showed that the polymorphism in this region is globally identical to that of the leader peptide sequences (data not shown). However, in the 5 -untranslated region, a sequence of $\sim 20$ nucleotides located immediately upstream of the start codon (ATG) was less polymorphic. Therefore, we designed multiple 5 ' PCR primers (18-mers) that terminated with the ATG sequence. This ensured a perfect match of the $3^{\prime}$ end of each primer with the template, which was shown to be crucial to initiate consistent priming of the Taq polymerase extension reaction. A Sall restriction site and four additional nucleotides were added to the $5^{\prime}$ end of the primers to facilitate cloning of the PCR fragments. Complex sets of $5^{\prime}$ primers were synthesized consisting of two primers for $\lambda, 30$ primers for $\kappa$, and 42 primers for the heavy-chain genes, some of which contained inosine residues or were degenerate (Fig. 2).

In contrast with the $5^{\prime}$-untranslated region, alignments of the $5^{\prime}$ end of the intron sequences downstream of the $J$ segments did not reveal significant polymorphism. Moreover, alignments showed that 3' PCR primers specific for each of the $J_{\lambda}, J_{\kappa}$, and $J_{H}$ segments could be designed in this intronic region. For this reason, a single PCR primer was prepared downstream of each J segment, except for the $\mathrm{J}_{\mathrm{H} 3}$ segment, where two primers were needed because of DNA sequence polymorphism. The $3^{\prime}$ PCR primers were 18-mers, except for the primer downstream of the $\mathrm{J}_{\mathrm{H} 1}$ segment which was a 20-mer, reflecting a high content of A/T nucleotides. A NotI restriction site and 4 additional nucleotides were added to the $5^{\prime}$ end of these oligonucleotides to allow directional cloning of the PCR fragments into expression vectors (Fig. 2).

To ensure specificity of the PCR amplifications and to characterize better the $V_{H}-D-J_{H}$ and $V_{L}-J_{L}$ rearrangements, sets of oligonucleotides (19-mers) specific for each of the $J_{\lambda}, J_{\kappa}$, and $J_{H}$ segments, which display a very low level of polymorphism, were synthesized. These were used as probes in both Southern blot analysis of the PCR fragments and Northern blots of total RNA extracted from the mouse hybridoma cells.

\section{Northern Blot Analysis of RNA from Mouse Hybridoma Cells Using J Segment-specific Oligonucleotide Probes}

The specificity of the oligonucleotides that hybridize to each of the J segments was demonstrated by Southern blot using a variety of cloned $V_{L}$ and $V_{H}$ PCR fragments of known sequences (data not shown). The specificity of these probes was also shown with Northern blots. RNA from the hybridoma cells 3G3 [IgM $(\lambda)]$ and HNK-20 [IgA ( $)$ ] were hybridized with the $J_{\lambda}, J_{\kappa}$, and $J_{H}$ oligonucleotides (Fig. 3). Probes $J_{\lambda 1}, J_{\kappa 2}$, and $J_{H 3}$ generated signals at the expected size for $3 \mathrm{G} 3$ and HNK-20, respectively. The signal generated by hybridization of HNK20 RNA with the $J_{\kappa 2}$ probe was much stronger than with the $\mathrm{J}_{\mathrm{H} 3}$ probe, although these two probes had similar specific activities and identical calculated melting temperatures. This observation suggests that either two or even three к-chain transcripts hybridized with the

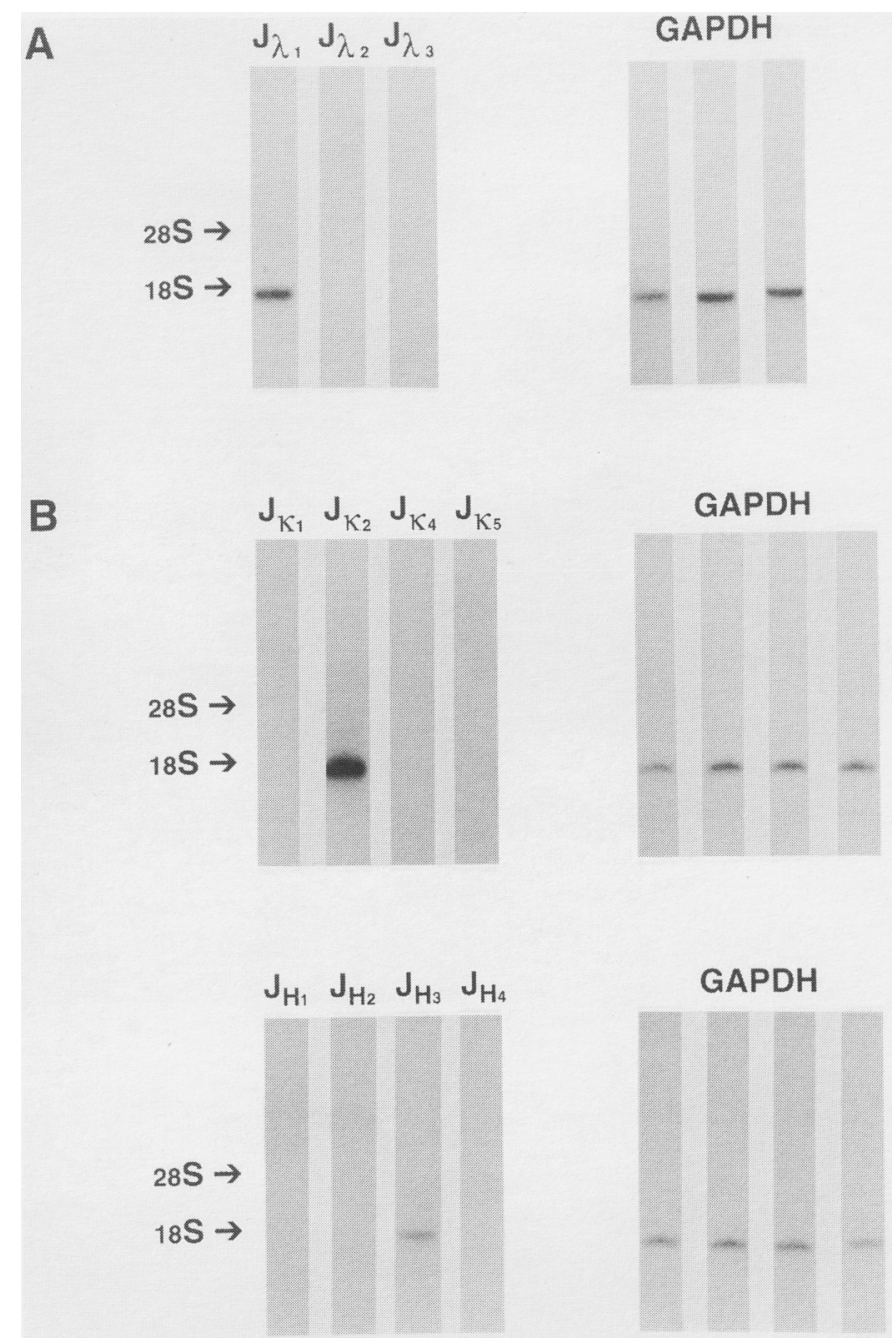

FIGURE 3 Northern blots of RNA isolated from hybridoma cells $3 G 3$ and HNK-20 probed with oligonucleotides homologous to the $\mathrm{J}_{\lambda}, \mathrm{J}_{\mathrm{\kappa}}$, and $\mathrm{J}_{\mathrm{H}}$ segments. $(A)$ Total RNA (10 $\mu \mathrm{g}$ per lane) from $3 \mathrm{G} 3[\operatorname{IgM}(\lambda)]$ was hybridized with the oligonucleotides $\mathrm{J}_{\lambda 1}, \mathrm{~J}_{\lambda 2}$, and $\mathrm{J}_{\lambda 3}$. (B) Total RNA (10 $\mu$ g per lane) from HNK-20 [IgA ( $\kappa$ )] was hybridized with $\mathrm{J}_{\kappa 1}, \mathrm{~J}_{\kappa 2}, \mathrm{~J}_{\kappa 4}$, and $\mathrm{J}_{\kappa 5}$, or with $\mathrm{J}_{\mathrm{H} 1}, \mathrm{~J}_{\mathrm{H} 2}, \mathrm{~J}_{\mathrm{H} 3}$, and $\mathrm{J}_{\mathrm{H} 4}$ (see Fig. 2 for sequences of the oligonucleotide probes). The oligonucleotides specific for the pseudogenes $\mathrm{J}_{\mathrm{K} 3}$ and $\mathrm{J}_{\lambda 4}$ were not used in this experiment. As an internal control for total RNA loading, Northern blots were stripped and rehybridized with a full-length glyceraldehyde-3-phosphate-dehydrogenase (GAPDH) cDNA probe. ${ }^{(18)}$ Positions of $18 \mathrm{~S}$ and $28 \mathrm{~S}$ rRNAs are indicated. 
$\mathrm{J}_{\mathrm{k} 2}$ probe. One of these transcripts, corresponding to a pseudogene rearranged with the $\mathrm{J}_{\mathrm{k} 2}$ segment, originated from the immortalizing fusion partner X63Ag8.653. ${ }^{(28,29)}$ For this reason, at least one $\kappa$ pseudogene transcript and one $\kappa$ functional gene transcript, both of them rearranged with the $J_{\kappa 2}$ segment, contributed to the strong signal observed. PCR amplification of the $V_{k}$ genes from HNK-20 genomic DNA supports the hypothesis that three $\kappa$-chain genes were rearranged with the $\mathrm{J}_{\mathrm{k} 2}$ segment. All three of these rearrangements were transcribed using reverse transcriptase-PCR (RT-PCR) (data not shown). Consequently, the signal observed on Northern blots hybridized with the $J_{\kappa 2}$ oligonucleotide probe is composed of three superimposed sig- nals. In all cases, the Northern blots were rehybridized with a probe specific for the housekeeping enzyme gene glyceraldehyde-3-phosphate-dehydrogenase ${ }^{(25)}$ to control for the amount of RNA loaded (Fig. 3).

\section{Strategy for Mouse $V_{H}$ and $V_{L} P C R$ Amplification}

Several immunoglobulin variable region genes were amplified by PCR using genomic DNA prepared from mouse hybridoma cells. As an example, we describe the amplification of $V_{\lambda}$ of the $3 G 3$ hybridoma, $V_{\kappa}$, and $V_{H}$ regions of the HNK-20 hybridoma.

From the Northern blot data shown in Figure 3, it was established that the $\lambda$ chain gene was rearranged with $J_{\lambda 1}$, the $k$ chain genes with $\mathrm{J}_{\kappa 2}$, and the heavychain gene with $\mathrm{J}_{\mathbf{H 3}}$. Therefore, the 3' primers required for amplification were $3^{\prime} \lambda 1,3^{\prime} \kappa 2$, and $3^{\prime} \mathrm{H} 3 \mathrm{a}$ or $3^{\prime} \mathrm{H} 3 \mathrm{~b}$, respectively. Because of the high degree of polymorphism seen in the 5'-untranslated region, all 5' primers had to be included in the PCR reactions in combination with these $3^{\prime}$ primers. This would result in two PCR reactions for $\lambda, 30$ for $\kappa$, and 42 for the heavy-chain genes, if each PCR reaction contained one $5^{\prime}$ primer and one $3^{\prime}$ primer. To reduce the number of combinations of $5^{\prime}$ and $3^{\prime}$ primers and, consequently, the number of PCR reactions, the $5^{\prime}$ primers were divided into small groups of 2,3 , or 4 primers based on their melting temperature (Fig. 2). This resulted in 1 group for $\lambda$ (group a), 9 for $\kappa$ (groups a-i), and 12

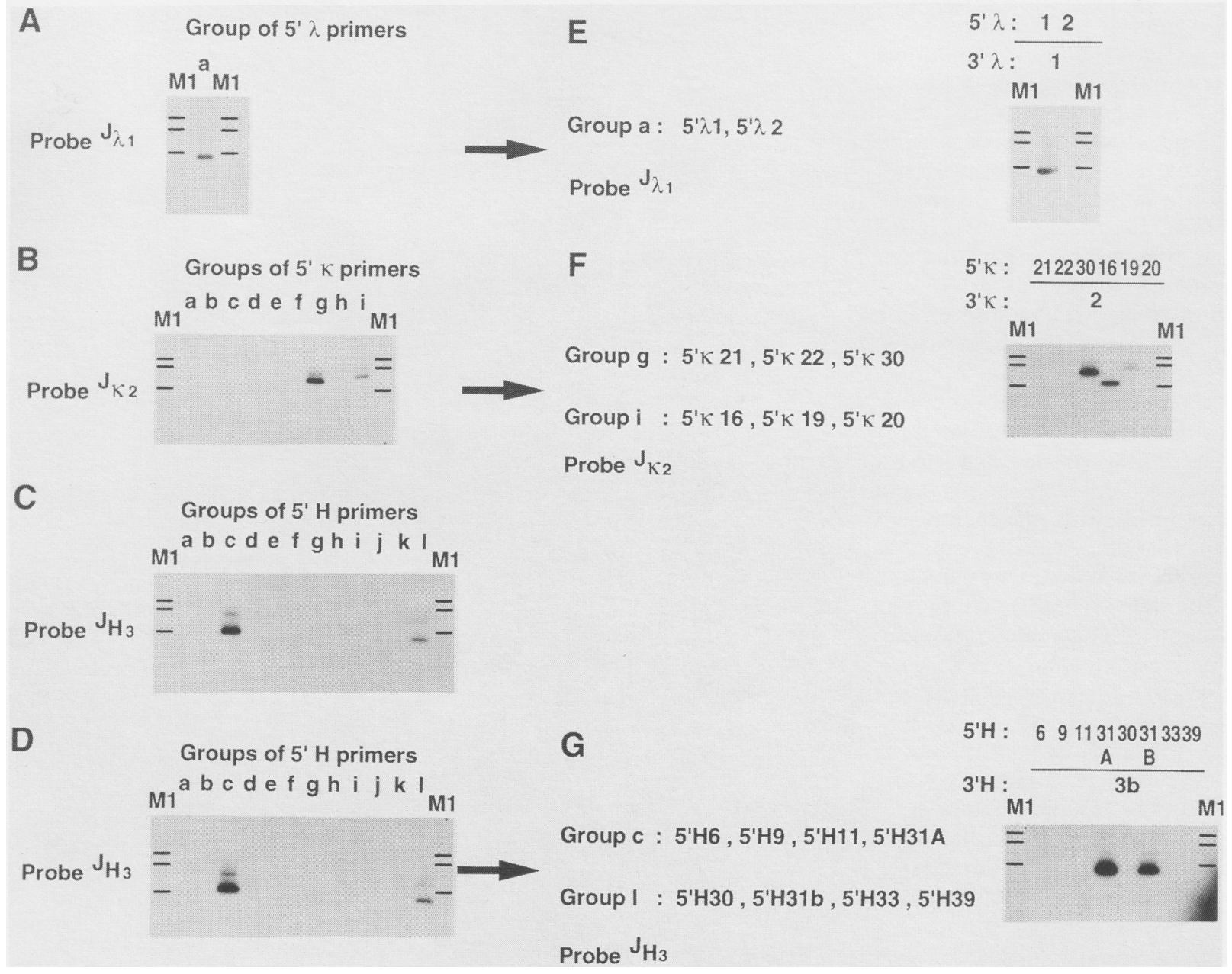

FIGURE 4 Southern blot analysis of the amplified $3 G 3 V_{\lambda}, H N K-20 V_{k \prime}$ and $V_{H}$ genomic fragments following a two-step PCR amplification. In the first step $(A-D)$, PCR amplifications were performed with groups of $5^{\prime}$ primers (see Fig. 2) combined with a single $3^{\prime}$ primer. The $3^{\prime}$ primer for $V_{\lambda}$ was $3^{\prime} \lambda 1$; for $\mathrm{V}_{\mathrm{k}} 3^{\prime} \mathrm{k} 2$; and for $\mathrm{V}_{\mathrm{H}} 3^{\prime} \mathrm{H} 3 \mathrm{a}$ or $3^{\prime} \mathrm{H} 3 \mathrm{~b}$. In the second step $(E-G)$, PCR amplifications were performed separately with each of the $5^{\prime}$ primers from the positive groups, allowing the $5^{\prime}$ primers that generate the signal in the first reaction to be identified. PCR amplification with $3^{\prime} \mathrm{H} 3 \mathrm{a}$ was omitted in the second step, as $3^{\prime} \mathrm{H} 3 \mathrm{~b}$ generates stronger signals. Positions of size markers of 564, 831, and 947 bp are indicated (lane M1). 
for the heavy-chain gene primers (groups a-1). A first set of PCR reactions contained one group of $5^{\prime}$ primers and one $3^{\prime}$ primer, thereby reducing the number of PCR reactions to 1 for $V_{\lambda}, 9$ for $V_{k}$, and 12 for $V_{H}$. Southern blot analyses of these PCR reactions, using the $\mathrm{J}_{\lambda 1^{-}}, \mathrm{J}_{\mathrm{K}^{-}}$, and $\mathrm{J}_{\mathrm{H}^{3}}$-specific oligonucleotide probes, allowed the identification of the combinations that generated positive signals: $5^{\prime} \lambda$ group a with $3^{\prime} \lambda 1 ; 5^{\prime} \kappa$ groups $g$ and $i$ with $3^{\prime} \kappa 2$; and $5^{\prime}$ heavy-chain groups $c$ and 1 with $3^{\prime} \mathrm{H} 3 \mathrm{a}$ or $3^{\prime} \mathrm{H} 3 \mathrm{~b}$ (Fig. 4A-D). These combinations were reanalyzed in a second set of PCR reactions that each contained one $5^{\prime}$ and one $3^{\prime}$ primer, to identify the pairs of primers that generated positive signals. These PCR reactions were analyzed again by Southern blot using the $\mathrm{J}_{\lambda 1}, \mathrm{~J}_{\mathrm{K} 2}$, and $\mathrm{J}_{\mathrm{H} 3}$ oligonucleotide probes. Results showed that the positive signals were generated by the following combinations: $5^{\prime} \lambda 1$ with $3^{\prime} \lambda 1$ (Fig. $4 \mathrm{E}$ ); $5^{\prime} \kappa 30,5^{\prime} \kappa 16$, or $5^{\prime} \kappa 19$ with $3^{\prime} \kappa 2$ (Fig. 4F); and $5^{\prime} \mathrm{H} 31 \mathrm{~A}$ or $5^{\prime} \mathrm{H} 31 \mathrm{~B}$ (which have very closely related sequences) with $3^{\prime} \mathrm{H} 3 \mathrm{~b}$ (Fig. 4G). These results indicate that one $\lambda$-chain gene was rearranged in $3 \mathrm{G} 3$, and three $\mathrm{k}$-chain genes and one heavy-chain gene were rearranged in HNK-20 (Fig. 5).

With another mouse hybridoma cell (PCG-4), secreting IgG2a with a $\kappa$ chain, three different rearrangements of the $\kappa$-chain genes, and a single rearrangement of the heavy-chain gene were also observed using the same PCR strategy (data not shown).

The presence of three $V_{k}$ rearrangements in HNK-20 and PCG-4 is consistent with both a functional $V_{\kappa}$ and an aberrant $V_{k}$ in all hybridoma cells obtained by fusion with cell lines derived from the original MOPC-21 tumor. ${ }^{(28,29)}$ A third $\kappa$-chain rearrangement was detected in both HNK-20 and PCG-4 hybridomas, although it involved different $J_{\kappa}$ segments. RT-PCR showed that this third rearrangement was transcribed in HNK-20 and silent in PCG-4 (data not shown). Because the only $\kappa$-chain gene that the fusion partner could have contributed is the nonfunctional MOPC-21 $\kappa$ gene, ${ }^{(30)}$ this third rearrangement probably originated from the mouse $B$ cell that served as a fusion partner. Contamination of the HNK-20 hybridoma cell by another hybridoma cell is improbable because a single heavy-chain rearrangement was detected in both Northern and Southern blots.
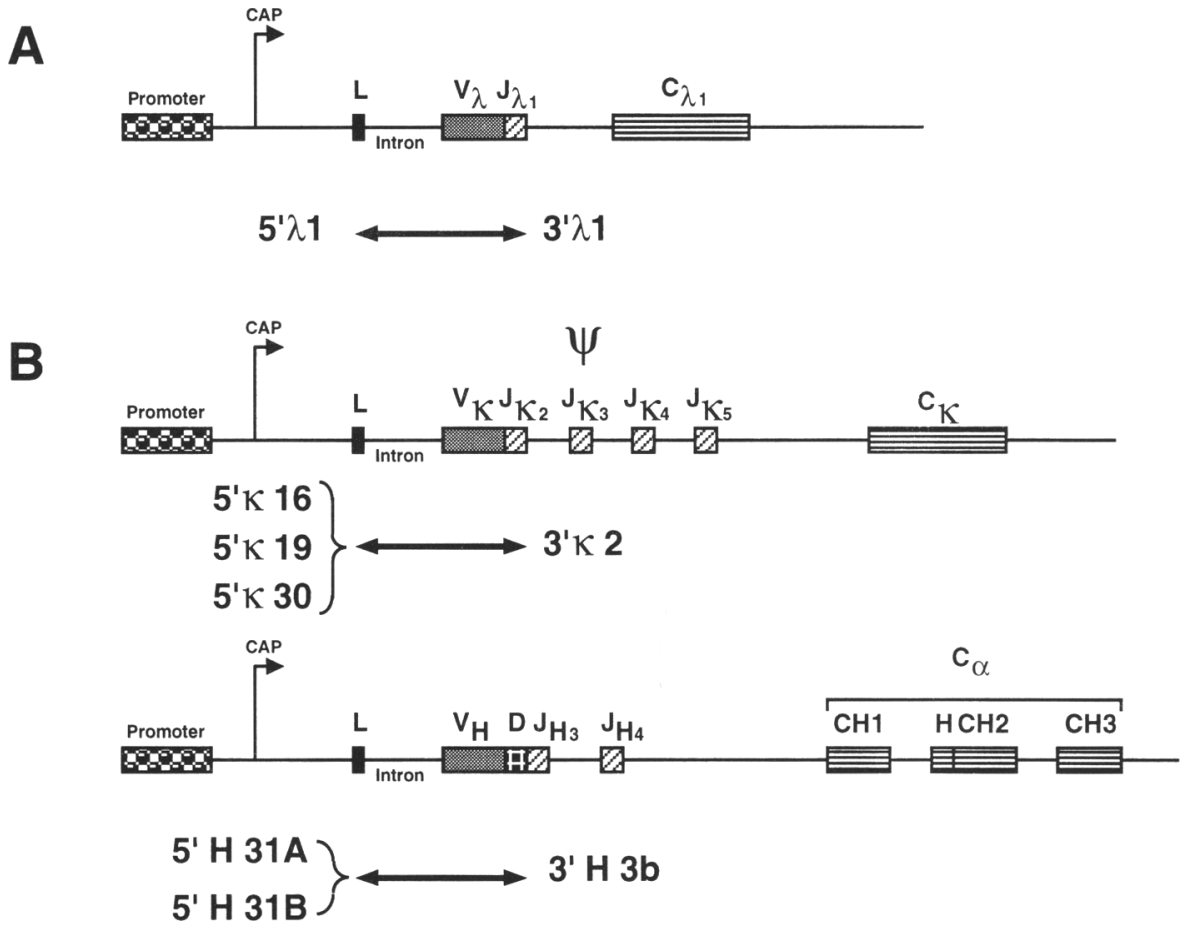

FIGURE 5 Map of the rearranged $3 G 3 V_{\lambda}$, and HNK-20 $V_{k}$, and $V_{H}$ regions. (A) Rearrangement of the $\lambda$ locus from 3G3; $(B)$ rearrangements of the $\kappa$ and heavy-chain loci from HNK-20. Three different $\kappa$-chain gene rearrangements were observed with the $J_{\kappa 2}$ segment. For the heavy-chain genes, 5' $\mathrm{H} 31 \mathrm{a}$ and $5^{\prime} \mathrm{H} 31 \mathrm{~b}$ generated the same PCR product; hence, a single rearrangement was observed for the heavy chain. The maps are not to scale. The pseudogene is indicated by $\Psi$.

\section{Sequence Analysis of the $V_{\lambda}, V_{\kappa^{\prime}}$ and $V_{H}$ PCR Fragments}

The PCR-amplified variable regions of $3 G 3 V_{\lambda}$, HNK-20 $V_{k}(s)$, and HNK-20 $V_{H}$ were cloned into the pING expression vector $^{(16)}$ and sequenced using the dideoxy chain termination technique. ${ }^{\text {(26) }}$ Sequences showed that the $3 G 3 V_{\lambda}\left(5^{\prime} \lambda 1-3^{\prime} \lambda 1\right)$, HNK-20 $V_{\kappa}\left(5^{\prime} \kappa 16-\right.$ $\left.3^{\prime} \kappa 2\right)$, HNK-20 $\mathrm{V}_{\kappa} \quad\left(5^{\prime} \kappa 19-3^{\prime} \kappa 2\right)$, and HNK-20 $V_{H}$ (5'H31B-3'H3b) fragments encode functional variable regions (Fig. $6 \mathrm{~A}-\mathrm{D})$. The sequence of the HNK-20 $\mathrm{V}_{\mathrm{\kappa}}$ $\left(5^{\prime} \kappa 19-3^{\prime} \kappa 2\right)$ fragment is not reported here because its coding sequence was identical to a published immunoglobulin $V_{\kappa}$ cDNA sequence. ${ }^{(31)}$ The deduced amino acid sequences of the functional $V_{\lambda}, V_{\kappa}$, and $V_{H}$ fragments correspond to open reading frames that are highly homologous to other mouse immunoglobulin variable regions. In these four PCR amplified fragments, the positions of the cysteines involved in the intramolecular disulfide bridge were conserved. The presence of conserved stretches of amino acids corresponding to framework sequences allowed the CDRs to be positioned (Fig. 6). Taken together, these structural features suggest that the amplified and cloned Fv fragments should be functional. However, it was not possible to determine by computer modeling which of the two $\kappa$ fragments, shown by sequencing to be functional, is bound to the HNK-20 $V_{H}$ (data not shown). Consequently, the two functional HNK$20 \mathrm{~V}_{\mathrm{K}} \mathrm{s}$ and the HNK-20 $\mathrm{V}_{\mathrm{H}}$ have been inserted into expression vectors containing human heavy- and light-chain constant genes and cotransfected into myeloma cells. Currently, the secreted chimeric antibodies are being tested for their ability to recognize their corresponding antigen.

The cDNA sequence of the HNK-20 $\mathrm{V}_{\kappa}$ nonfunctional rearranged gene has been reported $^{(28,29)}$ and was shown to contain a tyrosine instead of the invariant cysteine in position 23. Moreover, at the site of VJ recombination, 4 nucleotides appear to have been deleted, leading to a frame shift. This results in a premature termination codon at the 3 ' end of the $I$ segment at position 105 . We report here the genomic sequence of this $V_{\kappa}$ pseudogene (Fig. 6C) in which the cod- 
$\boldsymbol{A}$

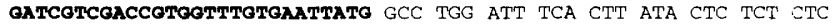
Met Ala Trp Ile ser Leu Ile Leu ser Leu

CTG GCT CTC AGC TCA G GTCAGCAGCCTTTCTACACTGCAGTGGGTATGCAACAATGCGCAT Leu Ala Leu Ser Ser G

CTTGTCTCTGATTTGCTACTGATGACTGGATTTCTCATCTGTTTGCAG GG GCC ATT TCC CAG

ly Ala Ile Ser Gln

GCT GTT GTG ACT CAG GAA TCT GCA CTC ACC ACA TCA CCT GGT GAA ACA GTC Ala Val Val Thr Gln Glu ser Ala Leu Thr Thr Ser pro Gly Glu Thr val

ACA CTC ACT TGT CGC TCA AGT ACT GGG GCT GTT ACA ACT AGT AAC TAT GCC Thr Leu Thr Cys Arg Ser Ser Thr Gly Ala Val Thr Thr Ser Asn Tyr Ala

AAC TGG GTC CAA GAA AAA CCA GAT CAT TTA TTC ACT GGT CTA ATA GGT GGT Asn Trp Val Gln Glu Lys pro Asp His Leu phe Thr Gly Leu Ile Gly gly

ACC AAC AAC CGA GCT CCA GGT GTT CCT GCC AGA TTC TCA GGC TCC CTG ATT Thr Asn Asn_arg Ala pro Gly Val pro Ala Arg phe ser Gly Ser Leu Ile

GGA GAC AAG GCT GCC CTC ACC ATC ACA GGG GCA CAG ACT GAG GAT GAG GCA Gly Asp Lys Ala Ala Leu Thr Ile Thr Gly Ala Gln Thr Glu Asp Glu Ala ATA TAT TTC TGT GCT CTA TGG TAC AGC AAC CAT TGG GTG TTC GGT GGA GGA Ile Tyr phe Cys Ala Leu Tro Tyr Ser Asn His Tro Val Phe Gly Gly Gly

ACC AAA CTG ACT GTC CTA G GTGAGTCACTCCTCCCTCCTTTGCGGCCGCTGAT Thr Lys Leu Thr Val Leu

oATcotconctrccagctctcagagAto GAG ACA GAC ACA CTC CTG TTA TGG GTA Met Glu Thr Asp Thr Leu Leu Leu Trp Val

CTG CTG CTC TGg GTT CCA G GTGAGAGTGCAGAGAaGTGTTGGATGCAACCTCTGTGGCCA Leu Leu Leu Trp Val Pro G

TTATGATACTCCATGCCTCTCTGTTCTTGATCACTATAATTAGGGCATTTGTCACTGGTTTTAAGTT TCCCCAGTCCCCTGAATTTTCCATTTCCTCAGAGTGATGTCCAAAATTCTTCTTAAAAATTTAAATC AAAAGGTCCTCTGCTGTGAAGTCTTTTATACATATATAACAATAATCTTTGTGTTTATCATTCCAG

GT TCC ACT GGT GAC ATT GTG CTG ACA CAG TCT CCT GCT TCC TTA GCT GTA ly Ser Thr Gly Asp Ile val Leu Thr Gln Ser Pro Ala ser Leu Ala val

TCT CTG GGG CAG AGG GCC ACC ATC TCA TAC AGG GCC AGC AAA AGT GTC AGT 416 Ser Leu Gly Gln Arg Ala Thr Ile Ser Tyr Arg Ala ser Lys ser Val ser 50

ACA TCT GGC TAT AGT TAT ATG CAC TGG AAC CAA CAG AAA CCA GGA CAG CCA 467 Thr ser Gly Tyr ser Tyr Met His Trp Asn Gin Gln Lys Pro Gly Gln Pro 67

CCC AGA CTC CTC ATC TAT CTT GTA TCC AAC CTA GAA TCT GGG GTC CCT GCC 518 Pro Arg Leu Leu Ile Tyr Leu Val Ser Asn Leu Glu Ser Gly Val pro Ala 84

AGG TTC AGT GGC AGT GGG TCT GGG ACA GAC TTC ACC CTC AAC ATC CAT CCT 569 Arg Phe Ser Gly Ser Gly Ser Gly Thr Asp Phe Thr Leu Asn Ile His Pro 101

GTG GAG GAG GAG GAT GCT GCA ACC TAT TAC TGT CAG CAC ATT AGG GAG CTT 620 Val Glu Glu Glu Asp Ala Ala Thr Tyr Tyr Cys Gln His Ile Arg Glu Leu 118

ACA CGT TCG GAG GGG GGA CCA AGC TGG AAA TAA AAC GTAAGTAGTCTTCTCAACT 675 Thr Arg Ser Glu Gly Gly Pro ser Trp Lys * *

FIGURE 6 Sequences of the PCR-amplified $3 G 3 V_{\lambda}$, HNK-20 $V_{\kappa}$, and $V_{H}$ regions. $(A) 3 G 3 V_{\lambda}$ fragment $\left(5^{\prime} \lambda 1-3^{\prime} \lambda 1\right)$; $(B)$ HNK-20 $V_{\kappa}$ fragment $\left(5^{\prime} \kappa 16\right.$ $\left.3^{\prime} \kappa 2\right)$; (C) HNK-20 $\mathrm{V}_{\kappa}$ (pseudogene) fragment $\left(5^{\prime}{ }^{\prime} 30-3^{\prime} \kappa 2\right) ;(D) \mathrm{HNK}-20 \mathrm{~V}_{\mathrm{H}}$ fragment $\left(5^{\prime} \mathrm{H} 31 \mathrm{~B}-3^{\prime} \mathrm{H} 3 \mathrm{~b}\right)$. These sequences have been deposited with the EMBL GenBank under accession numbers X82687 (3G3 $\left.V_{\lambda}\right), X 82688\left(H N K-20 V_{k}\right)$, X82689 (HNK-20 $V_{\kappa}$ pseudogene), and X82690 (HNK-20 $\left.V_{H}\right)$. The sequence of the PCR primers is shown in boldface type, the CDR regions are underlined, and the stop codon is noted by stars. Regions homologous to the $\mathrm{J}_{\lambda}, \mathrm{J}_{\kappa}$, and $\mathrm{J}_{\mathrm{H}}$ oligonucleotide probes are double underlined. 
ing sequence is in complete agreement with the reported cDNA sequences. This observation showed that the $V_{k}$ pseudogene was not affected by recombination events, at least in its coding region, during the PCR amplification process. Such PCR artifacts generating hybrid DNA molecules previously have been shown to occur when amplifying target sequences that are members of a gene family. ${ }^{(32)}$ If the sequences of the PCR fragment and the corresponding CDNA or genomic clone, prepared without amplification techniques, are available, these artifacts can be ruled out. Consequently, because these sequences are not available for the functional $V_{\lambda}, V_{k}$, and $V_{H}$ PCR fragments shown in Figure 6, the possibility that they are hybrid molecules cannot be excluded. However, this hypothesis will be indirectly evaluated by comparison of the specificity and affinity of the original mouse antibody and the chimeric mouse-human antibodies.

In conclusion, we have designed a novel strategy that allows the cloning of the full-length, rearranged variable regions of mouse immunoglobulin genes. This strategy requires a small number of PCR amplifications; however, the large number of $5^{\prime}$ primers and the specificity of both $5^{\prime}$ and $3^{\prime}$ primers greatly increase the probability of amplifying the rearranged $V_{\lambda}, V_{k}$, and $V_{H}$ region genes. In addition, the amplified Fv genes can easily be inserted into expression vectors containing murine or human light- and heavy-chain constant genes into intron sequences. Thus, a serious impediment for the rapid construction of isotypeswitched and chimerized mouse immunoglobulins has been overcome.

\section{ACKNOWLEDGMENTS}

We thank Ms. Monique Reinhardt for her excellent technical assistance and Dr. Claude Bonnard for his contribution to DNA sequence analysis and comparisons on a VAX computer. We are grateful to Dr. Nicolas Fasel for his advice and suggestions. This work was supported by a research contract from OraVax, Inc. (Cambridge, MA 02139) and by the Swiss Priority Program for Biotechnology (grant 5002-34603).

\section{REFERENCES}

1. Khazaeli, M.B., M.N. Saleh, R.H. Wheeler, W.J. Huster, H. Holden, R. Carrano, and A.F. LoBuglio. 1988. Phase I trial of mul- tiple large doses of murine monoclonal antibody CO17-1A. II. Pharmacokinetics and immune response. J. Natl. Cancer Inst. 80: $937-942$.

2. Morrison, S.L., M.J. Johnson, L.A. Herzenberg, and V.T. Oi. 1984. Chimeric human antibody molecules: Mouse antigen-binding domains with human constant region domains. Proc. Natl. Acad. Sci. 81: 68516855.

3. Boulianne, G.L., N. Hozumi, and M.J. Shulman. 1984. Production of functional chimeric mouse/human antibody. Nature 312: 643-646.

4. Jones, P.T., P.H. Dear, J. Foote, M.S. Neuberger, and G. Winter. 1986. Replacing the complementary determining regions in a human antibody with those from a mouse. Nature 321: 522-525.

5. Co, M.S. and C. Queen. 1991. Humanized antibodies for therapy. Nature 351: 501502.

6. LoBuglio, A.F., R.H. Wheeler, J. Trang, A. Haynes, K. Rogers, E.B. Harvey, L. Sun, J. Ghrayeb, and M.B. Khazaeli. 1989. Mouse/human chimeric monoclonal antibody in man: Kinetics and immune response. Proc. Natl. Acad. Sci. 86: 4220-4224.

7. Isaacs, J.D., R.A. Watts, B.L. Hazleman, G. Hale, M.T. Keogan, S.P. Cobbold, and H. Waldmann. 1992. Humanized monoclonal antibody therapy for rheumatoid arthritis. Lancet 340: 748-752.

8. Jones, S.T. and M.M. Bendig. 1991. Rapid PCR-cloning of full-length mouse immunoglobulin variable regions. BioTechnology 9: 88-89.

9. Kettleborough, C.A., J. Saldanha, K.H. Ansell, and M.M. Bendig. 1993. Optimisation of primers for cloning libraries of mouse immunoglobulin genes using the polymerase chain reaction. Eur. J. Immunol. 23: 206-211.

10. Le Boeuf, R.D., F.S. Galin, S.K. Hollinger, S.C. Peiper, and J.E. Blalock. 1989. Cloning and sequencing of immunoglobulin variable region genes using degenerate oligonucleotides and polymerase chain reaction. Gene 82: 371-377.

11. Orlandi, R., D.H. Güssow, P.T. Jones, and G. Winter. 1989. Cloning immunoglobulin variable domains for expression by the polymerase chain reaction. Proc. Natl. Acad. Sci. 86: 3833-3837.

12. Gillies, S.D., K.-M. Lo, and J. Wesolowski. 1989. High level expression of chimeric antibodies using adapted cDNA variable region cassettes. I. Immunol. Methods 125: 191-202.

13. Liu, A.Y., P.W. Mack, C.I. Champion, and R.R. Robinson. 1987. Expression of mouse::human immunoglobulin heavy chain cDNA in lymphoid cells. Gene 54: $33-40$.

14. Gavilondo-Cowley, J.V., M.J. Coloma, J. Vazquez, M. Ayala, A. Macias, K.E. Fry, and J.W. Larrick. 1990. Specific amplifica- tion of rearranged immunoglobulin variable region genes from mouse hybridoma cells. Hybridoma 9: 407-417.

15. Leung, S., A.S. Dion, M.C. Pellegrini, D.M. Goldenberg, and H.J. Hansen. 1993. An extended primer set for PCR amplification of murine kappa variable regions. BioTechniques 15: 286-292.

16. Chothia, C., A.M. Lesk, A. Tramontano, M. Levitt, S.J. Smith-Gill, G. Air, S. Sheriff, E.A. Padlan, D. Davies, W.R. Tulip, P.M. Colman, S. Spinelli, P.M. Alzari, and R.J. Poljak. 1989. Conformations of immunoglobulin hypervariable regions. Nature 342: 877-883.

17. Kaluza, B., G. Betzl, H. Shao, T. Diamantstein, and U. Weidle. 1992. A general method for chimerization of monoclonal antibodies by inverse polymerase chain reaction which conserves authentic $\mathrm{N}$-terminal sequences. Gene 122: 321-328.

18. Ratech, H. and A. Masih. 1993. Sensitive detection of clonal antigen receptor gene rearrangements in non-Hodgkin's malignant lymphoma with an anchored polymerase chain reaction-based strategy. Am. J. Clin. Pathol. 100: 527-533.

19. Weissenhorn, W., E. Weiss, M. Schwirzke, B. Kaluza, and U. Weidle. 1991. Chimerization of antibodies by isolation of rearranged genomic variable regions by the polymerase chain reaction. Gene 106: 273-277.

20. Gross-Bellard, M., P. Oudet, and P. Chambon. 1973. Isolation of high molecular weight DNA from mammalian cells. Eur. J. Biochem. 36: 32-38.

21. Glisin, V., R. Crkvenjakov, and C. Byus. 1974. Ribonucleic acid isolated by cesium chloride centrifugation. Biochemistry 13: 2633-2637.

22. Chirgwin, J.M., A.E. Przybyla, R.J. MacDonald, and W.J. Rutter. 1979. Isolation of biologically active ribonucleic acid from sources enriched in ribonuclease. Biochemistry 18: 5294-5299.

23. McMaster, G.K. and G.G. Carmichael. 1977. Analysis of single- and doublestranded nucleic acids on polyacrylamide and agarose gels by using glyoxal and acridine orange. Proc. Natl. Acad. Sci. 74: 4835-4838.

24. Chomczynski, P. and P.K. Qasba. 1984. Alkaline transfer of DNA to plastic membrane. Biochem. Biophys. Res. Commun. 122: $340-344$.

25. Piechaczyk, M., J.M. Blanchard, L. Marty, C. Dany, F. Panabieres, S. El Sabouty, P. Fort, and P. Jeanteur. 1984. Post-transcriptional regulation of glyceraldehyde-3phosphate-deshydrogenase gene expression in rat tissues. Nucleic Acids Res. 12: 6951-6963.

26. Sanger, F., S. Nicklen, and A.R. Coulson. 1977. DNA sequencing with chain-terminating inhibitors. Proc. Natl. Acad. Sci. 74: 5463-5467. 
27. Kabat, E.A., T.T. Wu, H.M. Perry, K.S. Gottesman, and C. Foeller. 1991. "Sequences of proteins of immunological interest," 5th ed. U.S. Department of Health and Human Services, Washington, D.C.

28. Strohal, R., G. Kroemer, G. Wick, and R. Kofler. 1987. Complete variable region sequence of a nonfunctionally rearranged kappa light chain transcribed in the nonsecretor P3X63-Ag8.653 myeloma cell line. Nucleic Acids Res. 15: 2771.

29. Carroll, W., E. Mendel, and S. Levy. 1988. Hybridoma fusion cell lines contain an aberrant kappa transcript. Mol. Immunol. 25: 991-995.

30. Storb, A.B. and R. Wilson. 1980. Myeloma with multiple rearranged immunoglobulin $\kappa$ genes: Only one $\kappa$ gene codes for kappa chain. Nucleic Acids Res. 8: 46814687.

31. Sun, L.K., P. Curtis, E. Rakowicz-Szulczynska, J. Ghrayeb, N. Chang, S.L. Morrison, and $\mathrm{H}$. Koprowski. 1987. Chimeric antibody with human constant regions and mouse variable regions directed against carcinoma-associated antigen 17-1A. Proc. Natl. Acad. Sci. 84: 214-218.

32. Ford, J.E., M.G. McHeyzer-Williams, and M.R. Lieber. 1994. Chimeric molecules created by gene amplification interfere with the analysis of somatic hypermutation of murine immunoglobulin genes. Gene 142: 279-283.

Received September 28, 1994; accepted in revised form January 17, 1995. 


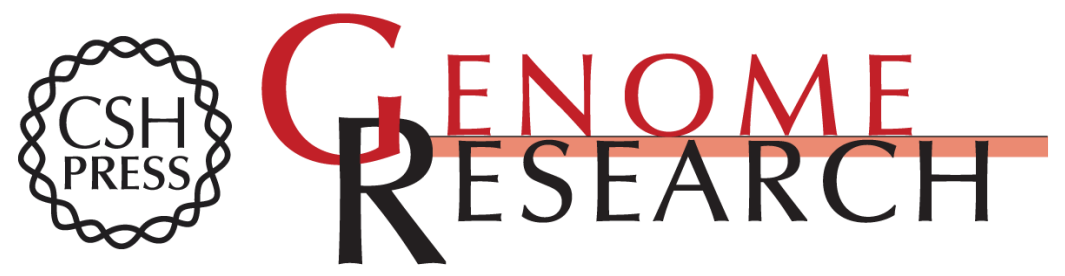

\section{Specific amplification by PCR of rearranged genomic variable regions of immunoglobulin genes from mouse hybridoma cells.}

J Berdoz, T P Monath and J P Kraehenbuhl

Genome Res. 1995 4: 256-264

References This article cites 31 articles, 6 of which can be accessed free at: http://genome.cshlp.org/content/4/5/256.full.html\#ref-list-1

License

Email Alerting

Receive free email alerts when new articles cite this article - sign up in the box at the Service top right corner of the article or click here.

\section{Affordable, Accurate Sequencing.}

To subscribe to Genome Research go to:

https://genome.cshlp.org/subscriptions 